\title{
Lagrangian model simulations of molecular mixing, including finite rate chemical reactions, in a temporally developing shear layer
}

\author{
Chester H. H. Chang and Werner J. A. Dahm \\ Department of Aerospace Engineering, The University of Michigan, Ann Arbor, Michigan 48109-2140 \\ Grétar Tryggvason \\ Department of Mechanical Engineering and Applied Mechanics, The University of Michigan, \\ Ann Arbor, Michigan 48109-2125
}

(Received 10 September 1990; accepted 22 January 1991)

\begin{abstract}
A local integral model for approximate simulations of the molecular mixing and chemical reaction processes in turbulent reacting flows is presented. The model is based on recent experimental results, which show that essentially all of the molecular mixing in turbulent flows occurs in thin strained laminar diffusion layers, and that the internal structure within these layers is essentially self-similar. This motivates a local integral treatment of the mixing and reaction processes in these layers that removes the resolution requirements imposed on full simulations by the steep gradients within the molecular diffusion and chemical reaction scales of the flow. The resulting integral model is applied to predict the mixing and reaction progress in a temporally developing shear layer over a range of Reynolds and Damköhler numbers, and to make comparisons with results obtained from full finite difference simulations. The resulting reactant and product concentration fields, as well as the temperature and reaction rate fields obtained from the model, are in good agreement with the full simulations. The results indicate that the model is able to accurately follow even highly sensitive nonlinear measures of the mixing and reaction progress such as the local extinction phenomenon characteristic of large Zel'dovich number Arrhenius kinetics.
\end{abstract}

\section{INTRODUCTION}

Full numerical simulations of turbulent flows, including ones in which molecular mixing and chemical reactions occur, have become possible over the past ten years. ${ }^{1-10}$ However, the need to computationally resolve the finest length scales on which gradients occur in the vorticity, species, and temperature fields limits such simulations of mixing and reacting flows, at present and well into the foreseeable future, to relatively low values of Reynolds, Schmidt, and Damköhler numbers. In particular, in fully turbulent flows, the competing effects of a local strain field $\sigma(t)$, which reduces the length scales on which vorticity gradients occur, and the diffusivity $v$, which acts to broaden the vorticity gradient scales, reach an equilibrium at a strain-limited vorticity diffusion length scale $\lambda_{v} \sim(v / \sigma)^{1 / 2}$. With the peak strain rates scaling as $\sigma \sim(u / \delta) \cdot \operatorname{Re}^{1 / 2}$, where $\operatorname{Re} \equiv(u \delta / v)$ is the local outer scale Reynolds number of the flow, the finest length scales arising in the vorticity field scale like $\lambda_{\nu} \sim \delta \cdot \mathrm{Re}^{-3 / 4}$. A similar balance ${ }^{11}$ between strain and diffusion of molecular species establishes the local strain-limited molecular diffusion scale $\lambda_{D} \sim(D / \sigma)^{1 / 2}$, and determines the finest length scale in the species concentration field as $\lambda_{D} \approx \lambda_{v} \cdot \mathrm{Sc}^{-1 / 2}$, where the Schmidt number Sc $\equiv(v / D)$ gives the ratio of vorticity and species diffusivities. Finally, embedded within this molecular diffusion scale is a reaction scale within which the chemical reactions occur, having a characteristic length scale ${ }^{12} \quad \lambda_{R} \sim \lambda_{D} \cdot \mathrm{Da}^{-1 / 3}$, where $\mathrm{Da} \equiv(k / \sigma)$ is the local Damköhler number with $k$ the specific rcaction rate constant. This hierarchy of increasingly finer length scales as $\mathrm{Re}, \mathrm{Sc}$, and $\mathrm{Da}$ are increased quickly drives such full simulations beyond the resolution capabilities of any practical calculation.

In view of the rather severe resolution restrictions introduced by the length scales $\lambda_{v}, \lambda_{D}$, and $\lambda_{R}$, numerous models based on temporal or spatial averages of the full equations governing the evolution of the flow field, as well as the molecular mixing and chemical reaction processes occurring within the flow, have been developed to treat turbulent mixing and reacting flows. In recent years, such models have come under increasing criticism for the way in which they treat interactions between the large-scale and small-scale transport processes. To a large extent, this criticism applies to the whole class of gradient transport models commonly used in closing Reynolds averaged formulations, as well as to the subgrid models used in large eddy simulations of turbulent flows. The proper treatment of these fine scales is perhaps most critical in flows that involve mixing and chemical reactions, since the actual reaction processes depend far more crucially on the instantaneous mixing field than on the average quantities.

Here we present a fundamentally different approach to the treatment of turbulent mixing and reacting flows that is more closely connected with the notion of "approximate simulations" than with classical modeling of the type described above. In particular, the model aims to alleviate the resolution requirements imposed on full simulations by the molecular diffusion scale $\lambda_{D}$ and the reaction scale $\lambda_{R}$. The method presented is based on recent experimental results, ${ }^{13-16}$ which show that essentially all the molecular mixing in turbulent flows at $\mathrm{Sc}>1$, as well as at $\mathrm{Sc} \approx 1$, occurs in thin strained laminar diffusion layers, and that the 
internal structure within these molecular diffusion layers is essentially self-similar. This layer-like fine structure suggests a thin-layer parabolization of the equations governing the diffusion and reaction processes in a local Lagrangian frame moving with a point in such a layer. The self-similar internal structure observed within these layers then motivates a local integral treatment of the mixing and reaction processes within them. Integral methods are, of course, a commonly used approximation in thin-layer problems that display a degree of self-similarity. Perhaps the most familiar example is in laminar boundary layers, where a variety of integral techniques such as Pohlhausen's method are closely related to the model developed here. The approach we take leads to a set of integral moment equations for the local normal structure of the strained diffusion and reaction layer, in which the precise internal details of the layer structure affects the evolution of the moments only through integrals resulting from the reaction rate terms. The nature of these "reaction integrals," together with the experimentally observed self-similarity in the internal structure of the layers, suggests that these moment equations can be closed by modeling these profiles as self-similar families of shapes specified by any desired number of degrees of freedom. This approach removes the resolution restrictions imposed on full simulations by the steep gradients within the layers, and instead requires only the evolution of the material interface initially separating the reactants to be computed. The principal advantage of this method is that the thin-layer approximation on which it is based should become increasingly more accurate as $\mathrm{Re}, \mathrm{Sc}$, and $\mathrm{Da}$ are increased, namely in precisely the regime where full simulations become infeasible.

We introduced such an approach in Ref. 17 in a model of isolated and parallel interacting diffusion and reaction layers. Here we couple an integral model of this type with a flow field calculation to predict the reactant and product concentration fields, as well as the temperature and reaction rate fields, in a temporally developing shear layer over a range of $\mathrm{Re}$ and $\mathrm{Da}$. The results obtained are compared with full finite difference simulations to assess the validity of the model. In particular, these comparisons test the ability of the model to accurately follow even highly sensitive nonlinear measures of the mixing and reaction progress such as the local extinction phenomenon characteristic of large Zel'dovich number Arrhenius kinetics. It is worth pointing out that the present model, in view of its conceptual basis in terms of mixing and reactions resulting from a combination of isolated and overlapping strained laminar diffusion layers, builds on earlier models that involve such strained diffusion layers. Of notable relevance are the "coherent flame model" of Marble and Broadwell ${ }^{18}$ and various implementations of "flamelet models" by Gibson and Libby, ${ }^{19}$ Liew et al.,${ }^{20}$ and Peters and Williams. ${ }^{21,22}$ Also related is the strained diffusion layer component of the shear layer mixing and reaction models of Broadwell and Breidentha ${ }^{23}$ and Broadwell and Mungal, ${ }^{24}$ as weil as the jet mixing model of Broadwell. ${ }^{25}$ As is the case in these earlier models, we focus here on the major physical mechanisms governing the molecular mixing and chemical reaction processes, and disregard for the present time such secondary effects as variations in density and transport prop- erties, though these may be significant in certain specific applications.

The presentation is organized as follows. In Sec. II we formulate the general mixing and reaction problem and discuss the finite difference technique used here to obtain full simulations for comparisons with the model results. Section III gives an overview of the model formulation, and in Sec. IV we present comparisons of detailed model results for the mixing and reaction processes in a temporally developing shear layer with corresponding results from full finite difference simulations. Integral comparisons between the model and the full simulation results are given in Sec. V, and in Sec. VI we draw conclusions regarding the use of this model for treating molecular mixing and chemical reaction processes.

\section{PROBLEM FORMULATION}

Here we formulate the mixing and reaction problem in a two-dimensional temporally developing shear layer. The flow field underlying the molecular diffusion and chemical reaction processes satisfies the two-dimensional NavierStokes equations, which can be conveniently expressed in vorticity form as

$$
\frac{\partial \omega}{\partial t}+J(\psi, \omega)-\frac{1}{\operatorname{Re}} \nabla^{2} \omega=0 ; \quad \nabla^{2} \psi=-\omega,
$$

where $J(\psi, \omega) \equiv(\partial \psi / \partial y)(\partial \omega / \partial x)-(\partial \psi / \partial x)(\partial \omega / \partial y)$. As far as the molecular mixing and reaction chemistry are concerned, a sufficiently realistic approximation for our purposes here is to treat this as an overall, single-step, binary, irreversible reaction between two species $A$ and $B$ to yield a product $P$ as

$$
v_{A} A+v_{B} B \stackrel{k}{\rightarrow} v_{P} P
$$

where $v_{i}$ are the molar stoichiometric coefficients. The overall reaction rate is taken to be first order with respect to each of the reactants, and the specific reaction rate constant $k$ is taken to be controlled by temperature-dependent Arrhenius kinetics of the form

$$
k=\mathrm{Ze}^{-E / R T},
$$

where $Z$ is the frequency factor and $E, R$, and $T$ are the overall activation energy, the universal gas constant, and the absolute temperature, respectively. The resulting advectiondiffusion-reaction equations for the molar concentrations $c_{i}$ of each of the species $i=\{A, B, P\}$ and the temperature $T$ are then $^{26}$

$$
\frac{\partial c_{i}}{\partial t}+J\left(\psi, c_{i}\right)-\frac{1}{\operatorname{ReSc}} \nabla^{2} c_{i}= \pm v_{i} \mathrm{Da}\left(c_{A} c_{B}\right) e^{-\left(\mathrm{Z}_{\mathrm{e}} / T\right)}
$$

$$
\frac{\partial T}{\partial t}+J(\psi, T)-\frac{1}{\operatorname{Re} \operatorname{Pr}} \nabla^{2} T=v_{P} \operatorname{He} \mathrm{Da}\left(c_{A} c_{B}\right) e^{-\left(\mathrm{ze}_{\mathrm{e}} / T\right)},
$$

with $J$ defined as above. The minus sign is appropriate in $\mathrm{Eq}$. (2a) when $i=\{A, B\}$ and the plus sign when $i=\{P\}$.

Note that in Eqs. (1) and (2) we have for simplicity assumed variations in the density and all transporl properties to be sufficiently small that they can be neglected 


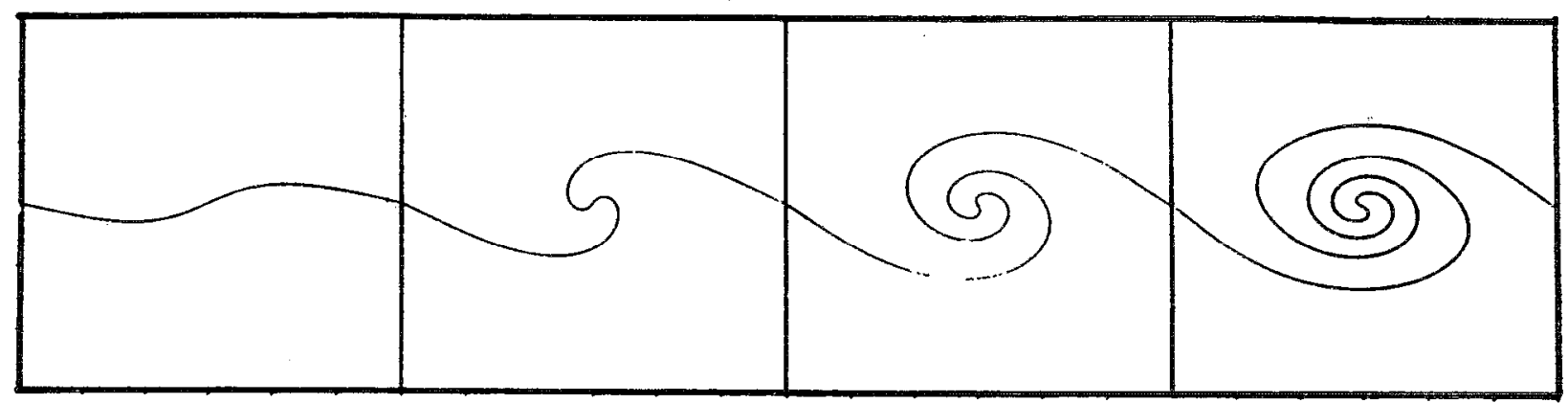

FIG. 1. The evolution of the material surface initially separating the two reactant streams in a temporally developing shear layer obtained from a full finite difference simulation of Eq. (1) at $\operatorname{Re}=2000$. Development of this surface is the only input to the Lagrangian mixing and reaction model.

(though we believe the model can be readily extended to the general case). All quantities have been made nondimensional by the reference scales $L^{*}, U^{*}, c^{*}$, and $T^{*}$ for the length, velocity, concentrations, and temperature, respectively, with the resulting dimensionless parameters given by

$$
\begin{aligned}
& \operatorname{ReSc}_{i} \equiv \frac{U^{*} L^{*}}{D_{i}} ; \quad \operatorname{Re} \operatorname{Pr} \equiv \frac{U^{*} L^{*}}{D_{T}} ; \\
& \mathrm{Da} \equiv \frac{Z c^{*}}{U^{*} / L^{*}} ; \quad \mathrm{Ze} \equiv \frac{E}{R T^{*}} ; \quad \mathrm{He} \equiv \frac{q}{c_{v} T^{*}},
\end{aligned}
$$

where $q$ is the heat release per unit mass of fuel and $c_{v}$ is the constant volume specific heat. Here $\mathrm{Da}$ is the Damköhler number, $\mathrm{Ze}$ is the Zel'dovich number, and $\mathrm{He}$ is the heat release parameter.

We can, of course, solve Eqs. (1), (2a), and (2b) directly by any of a number of numerical techniques to obtain a full simulation, though such simulations will be subject to the restrictions on $\mathrm{Re}, \mathrm{Sc}$, and $\mathrm{Da}$ introduced by the diffusion and reaction length scales noted in Sec. I. Here we conduct such full simulations at the relatively low parameter values accessible within these resolution limitations in order to allow detailed comparisons with the results obtained from the mixing and reaction model. In these full simulations, we use a finite difference method that is second order both in space and time. The spatial differencing uses centered differences with Arakawa's differencing of the Jacobian, and a predictor-corrector time integration is used. The computational domain is square and periodic in the horizontal direction with stress-free no flowthrough top and bottom boundaries. All of the calculations were performed on a $128^{2}$ grid, and were checked on a $64^{2}$ grid to confirm convergence. Note that the resulting full simulation of the flow field from Eq. (1) also gives the roll-up of the material surface that initially separated the two reactant streams $A$ and $B$. An example of this is shown in Fig. 1. This material surface, and, in particular, the distribution of strain rates along the surface, then provides the input to the molecular diffusion and chemical reaction model, as outlined below.

\section{MODEL FORMULATION}

In this section we give a brief overview of a local integral model used here to approximate the molecular mixing and chemical reaction processes represented by Eqs. (2a) and (2b). We begin with the observations in Refs. 13-16 that virtually all the molecular mixing in turbulent flows, at $\mathrm{Sc} \gg 1$ and at $\mathrm{Sc} \approx 1$, occurs in thin strained laminar diffusion layers. As a consequence, in a local Lagrangian coordinate frame moving with such a diffusion layer and remaining aligned with the layer, gradients within the layer will be small in comparison with the gradient normal to the layer. We therefore parabolize Eqs. (2a) and (2b) and introduce a local linear expansion for the velocity in the vicinity of the origin as

$$
\begin{aligned}
\frac{\partial c_{i}}{\partial t} & -\left(v_{n}+\sigma n\right) \frac{\partial c_{i}}{\partial n}-\frac{1}{\operatorname{ReSc_{i}}} \frac{\partial^{2} c_{i}}{\partial n^{2}} \\
& = \pm v_{i} \mathrm{Da}\left(c_{A} c_{B}\right) e^{-(\mathrm{Ze} / n}, \\
\frac{\partial T}{\partial t} & -\left(v_{n}+\sigma n\right) \frac{\partial T}{\partial n}-\frac{1}{\operatorname{Re} \operatorname{Pr}} \frac{\partial^{2} T}{\partial n^{2}} \\
& =v_{P} \mathrm{He} \mathrm{Da}\left(c_{A} c_{B}\right) e^{-(\mathrm{Ze} / T)}
\end{aligned}
$$

where $\sigma(t)$ is the normal component of the strain rate along the local interface-normal direction and $v_{n}$ is simply the uniform normal velocity required to keep the Lagrangian coordinate frame fixed to the reaction zone, as will be discussed below. Note that in Eqs. (3a) and (3b) we have also assumed noninertial terms resulting from the Lagrangian coordinate frame to be negligible.

\section{A. Local integral moments}

We define the local integral moments of each of the reactant gradient profiles, the product profile, and the temperature profile as

$$
\begin{aligned}
R_{j}^{l} & \equiv \int_{-\infty}^{+\infty} n^{j} \frac{\partial c_{i}}{\partial n} d n, \quad j=0,1,2, \ldots, \\
C_{j} & =\int_{-\infty}^{+\infty} n^{j} c_{p} d n, \quad j=0,1,2, \ldots, \\
Q_{j} & \equiv \int_{-\infty}^{+\infty} n^{j}\left(T-T_{\infty}\right) d n, \quad j=0,1,2, \ldots .
\end{aligned}
$$

From Eqs. (3a) and (3b) these moments must follow the transport equations

$\frac{d R_{0}^{i}}{d t}=0$ 


$$
\frac{d R_{1}^{i}}{d t}=-\sigma R_{1}^{i} \pm v_{n} c_{i}^{\infty}+v_{i} \mathrm{Da} \int_{-\infty}^{+\infty}\left(c_{A} c_{B}\right) e^{-(\mathrm{Ze} / T\rangle} d n
$$$$
\frac{d R_{2}^{i}}{d t}=-2 \sigma R_{2}^{i}-2 v_{n} R_{1}^{i} \mp 2 c_{i}^{\infty} \frac{1}{\operatorname{ReSc_{i}}}
$$$$
+2 v_{i} \mathrm{Da} \int_{-\infty}^{+\infty}\left(c_{A} c_{B}\right) e^{-(\mathrm{Ze} / n)} n d n,
$$$$
\frac{d C_{0}}{d t}=-\sigma C_{0}+v_{P} \mathrm{Da} \int_{-\infty}^{+\infty}\left(c_{A} c_{B}\right) e^{-(\mathrm{Ze} / T)} d n,
$$$$
\frac{d C_{1}}{d t}=-2 \sigma C_{1}-v_{n} C_{0}
$$$$
+v_{P} \mathrm{Da} \int_{-\infty}^{+\infty}\left(c_{A} c_{B}\right) e^{-(\mathrm{Ze} / T)} n d n
$$$$
\frac{d C_{2}}{d t}=-3 \sigma C_{2}-2 v_{n} C_{1}+2 C_{0} \frac{1}{\operatorname{ReSc_{P}}}
$$$$
+v_{P} \mathrm{Da} \int_{-\infty}^{+\infty}\left(c_{A} c_{B}\right) e^{-(\mathrm{Ze} / T)} n^{2} d n
$$

$$
\frac{d Q_{0}}{d t}=-\sigma Q_{0}+v_{P} \mathrm{He} \mathrm{Da} \int_{-\infty}^{+\infty}\left(c_{A} c_{B}\right) e^{-(\mathrm{Ze} / T)} d n,
$$$$
\frac{d Q_{1}}{d t}=-2 \sigma Q_{1}-v_{n} Q_{0}
$$$$
+v_{P} \mathrm{He} \mathrm{Da} \int_{-\infty}^{+\infty}\left(c_{A} c_{B}\right) e^{-(\mathrm{Ze} / T)} n d n
$$

$$
\begin{aligned}
\frac{d Q_{2}}{d t}= & -3 \sigma Q_{2}-2 v_{n} Q_{1}+2 Q_{0} \frac{1}{\operatorname{Re} \operatorname{Pr}} \\
& +v_{P} \mathrm{He} \mathrm{Da} \int_{-\infty}^{+\infty}\left(c_{A} c_{B}\right) e^{-(\mathrm{Ze} / T)} n^{2} d n,
\end{aligned}
$$

Note that in Eqs. (5)-(7), and from here on, the top sign corresponds to $i=\{A\}$ and the bottom sign to $i=\{B\}$. At this point we also choose $v_{n}$ by defining the origin of our Lagrangian frame so that $Q_{1} \equiv 0$ in Eq. (7b).

While these moment equations still involve the reactant and temperature profiles explicitly, the profiles enter only in the integrals resulting from the reaction rate terms. As a consequence, accurately tracking the evolution of the profile moments in Eqs. (4a)-(4c) via Eqs. (5) - (7) only requires that these reaction integrals be correctly approximated. The integral nature of these terms suggests this might be possible even with relatively crude assumptions for the actual profiles.

\section{B. Model profiles}

At this point we will make use of one further experimental observation in order to close the moment equations, namely the results in Refs. 13-15 that the internal structure within the molecular diffusion layers in turbulent flows is essentially self-similar. In view of this, we choose to specify the reactant and temperature profiles as a self-similar family of shapes parametrized by any chosen number of degrees of freedom. Note that once the family of profile shapes has been selected, the moment equations are closed and no further assumptions are necessary. Moreover, the nature of the reaction integrals suggests that the evolution equations are relatively insensitive to the specific choice of profile shapes. The results obtained in Ref. 17 suggest that this is true, even for remarkably crude model representations for these profiles. Here we implement the model with a relatively simple family of smooth "algebraic" profile shapes, given by

$$
\begin{aligned}
& c_{i}(n)=\frac{1}{2} c_{i}^{\infty}\left[1 \mp I\left(n \pm \Delta_{i} ; \delta_{i}\right)\right], \\
& c_{P}(n)=C_{M} J\left(n ; \delta_{P}\right), \\
& T(n)-T_{\infty}=T_{M} J\left(n ; \delta_{T}\right),
\end{aligned}
$$

where

$$
\begin{aligned}
& I\left(n ; \delta_{i}\right) \equiv \frac{n^{3}+3 / 2 \delta_{i}^{2} n}{\left(n^{2}+\delta_{i}^{2}\right)^{3 / 2}} \\
& J\left(n ; \delta_{i}\right) \equiv \frac{2}{3} \delta_{i} \frac{d I}{d n}=\frac{\delta_{i}^{5}}{\left(n^{2}+\delta_{i}^{2}\right)^{5 / 2}} .
\end{aligned}
$$

These model profiles are shown in Fig. 2, where $\Delta_{i}$ are the displacements of the reactant gradient profiles from the origin, $\delta_{i}$ are their widths, and $T_{M}$ and $C_{M}$ are, respectively, the peak temperature rise and product concentration across the layer. Each of the four profiles is thus parametrized by two
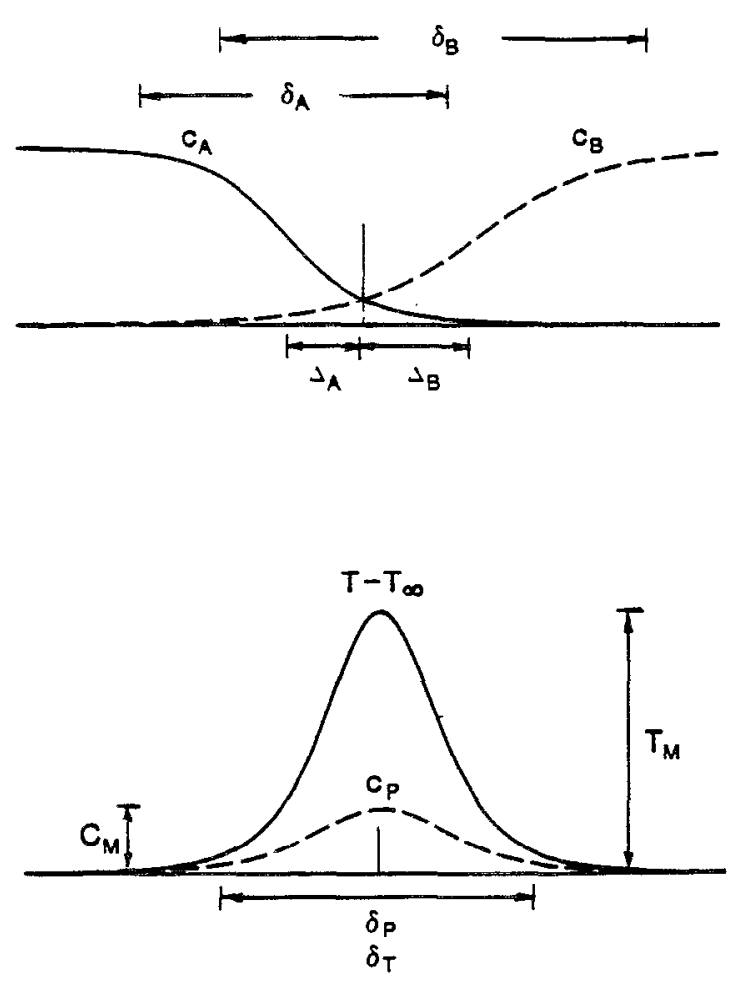

FIG. 2. The family of self-similar profile shapes used to model the internal structure within the diffusion and reaction layers in the model. 


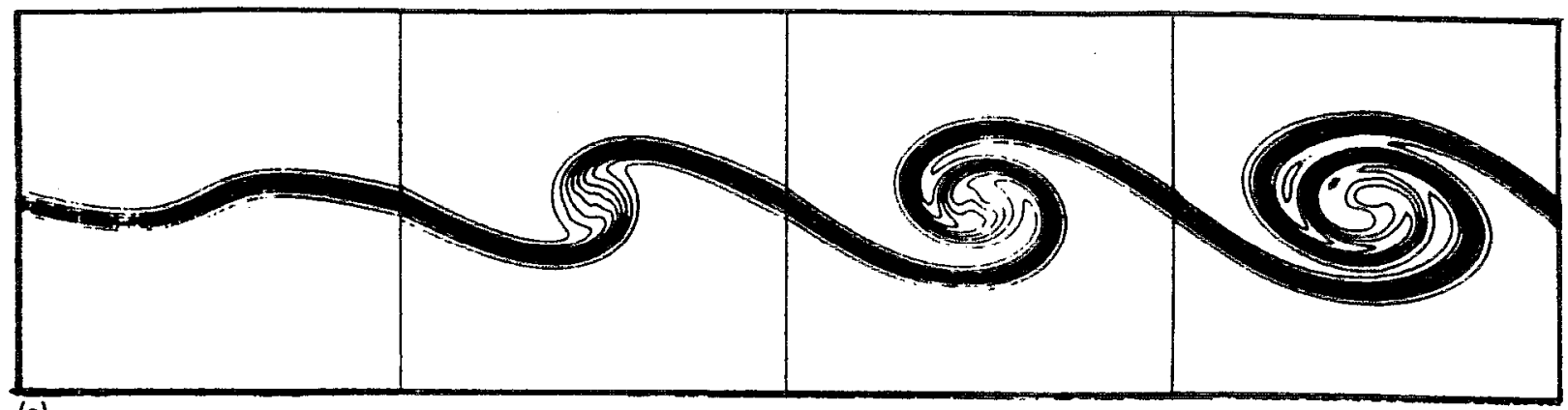

(a)

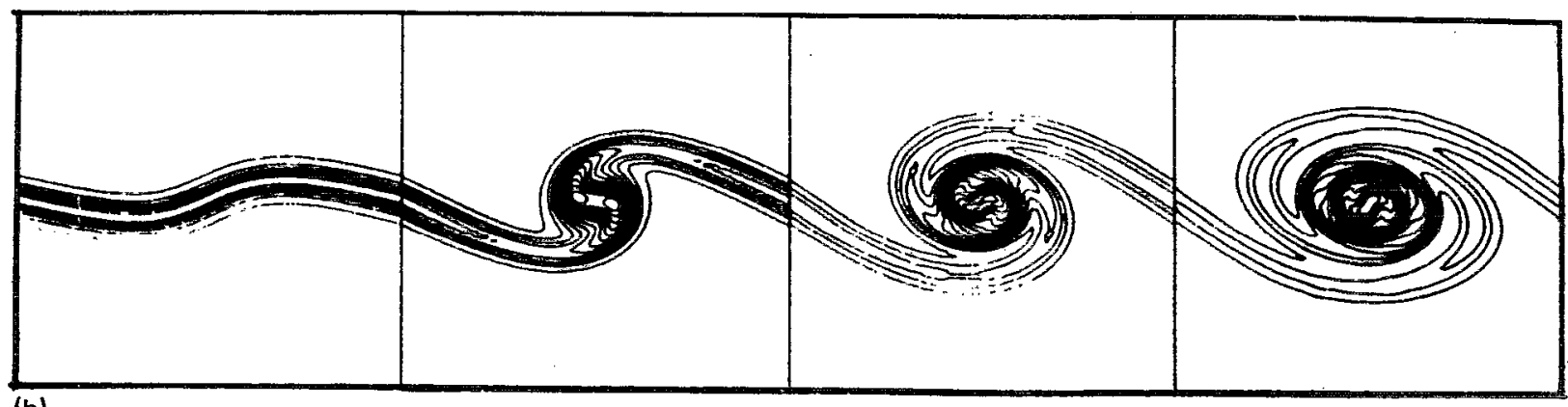

(b)

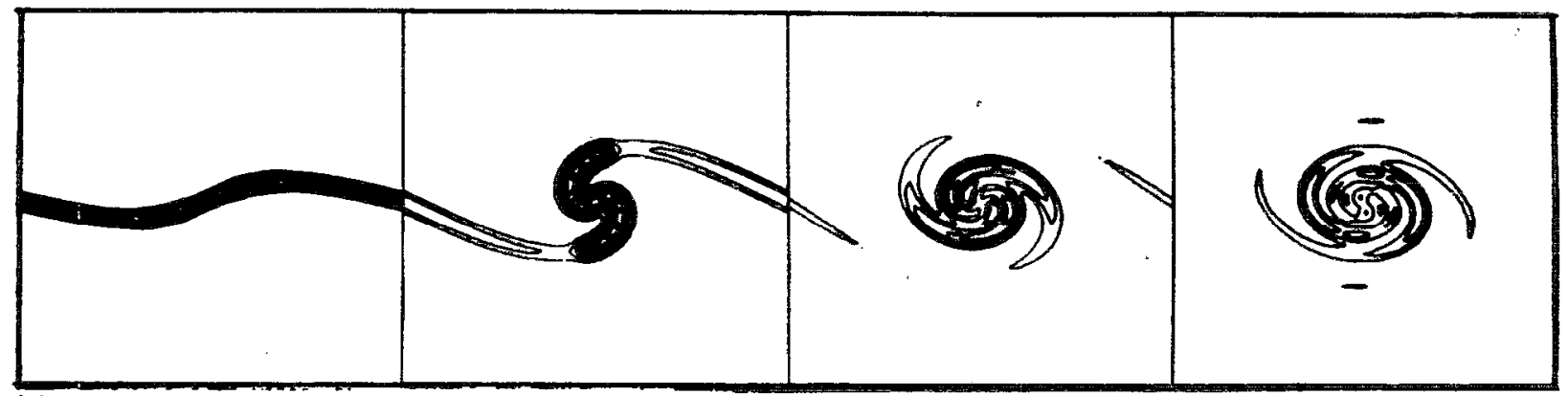

(c)

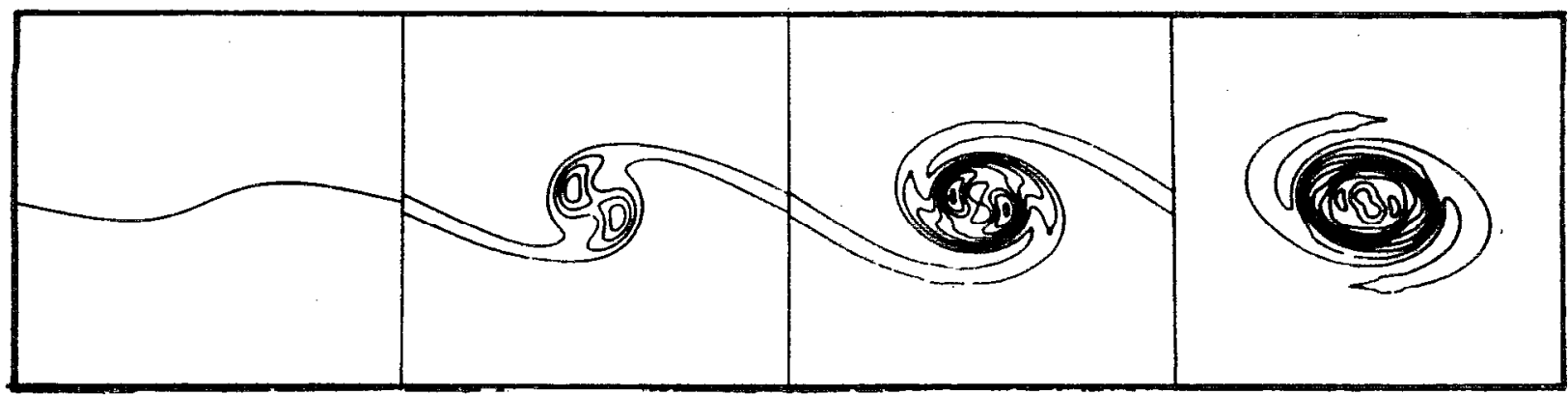

(d)

FIG. 3. Model results from Eqs. (5) - (10) for the interface roll-up given in Fig. 1, showing resulting reactant, temperature, reaction rate, and product fields at $\mathrm{Re}=2000$ and $\mathrm{Da}=30$. Compare with corresponding full finite difference results in Fig. 5. From left to right: $t=0,0.6,1.2$, and 1.8 . From top to bottom: (a) The scaled reactant concentration field $c_{, A}$, lowest contour level $=0.1$, contour interval $=0.1 ;$ (b) The scaled temperature rise field $T-T_{\text {. }}$, lowest contour level $=1.125$, contour interval $=0.250 ;$ (c) The scaled reaction rate field $\mathrm{Da}\left(c_{A} c_{B}\right) e^{-(z e / T)}$, lowest contour level $=0.03$, contour interval $=0.03$; (d) the scaled product concentration field $c_{P}$, lowest contour level $=0.02$, contour interval $=0.02$.

degrees of freedom, and accordingly the evolution of two moments for each profile must be followed with Eqs. (5)(7). The profile parameters are related to the evolving moments as

$$
\delta_{i}^{2}=-2\left[\left(R_{1}^{i} / c_{i}^{\infty}\right)^{2} \pm\left(R_{2}^{i} / c_{i}^{\infty}\right)\right] ; \quad \Delta_{i}=\left(R_{1}^{i} / c_{i}^{\infty}\right),
$$$$
C_{M}=\frac{3}{4}\left(C_{0}^{3} / 2 C_{2}\right)^{1 / 2} ; \quad \delta_{P}=\left(2 C_{2} / C_{0}\right)^{1 / 2},
$$

$$
T_{M}=\frac{3}{4}\left(Q_{0}^{3} / 2 Q_{2}\right)^{1 / 2} ; \quad \delta_{T}=\left(2 Q_{2} / Q_{0}\right)^{1 / 2} .
$$

Therefore the model involves tracking the reactant gradient moments $R_{1,2}^{i}$, the product moments $C_{0,2}$, and the temperature rise moments $Q_{0,2}$ everywhere along the evolving material surface that initially separated the two reactant streams using Eqs. (5)-(7). The strain rate distribution $\sigma(t)$ at each point on this deforming material surface drives the evolution 


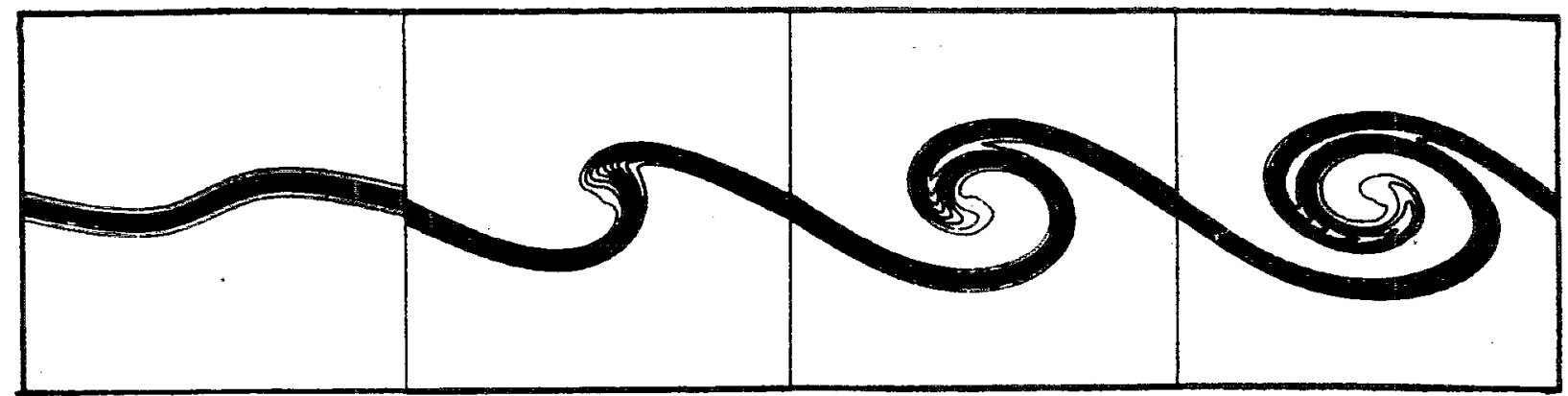

(a)

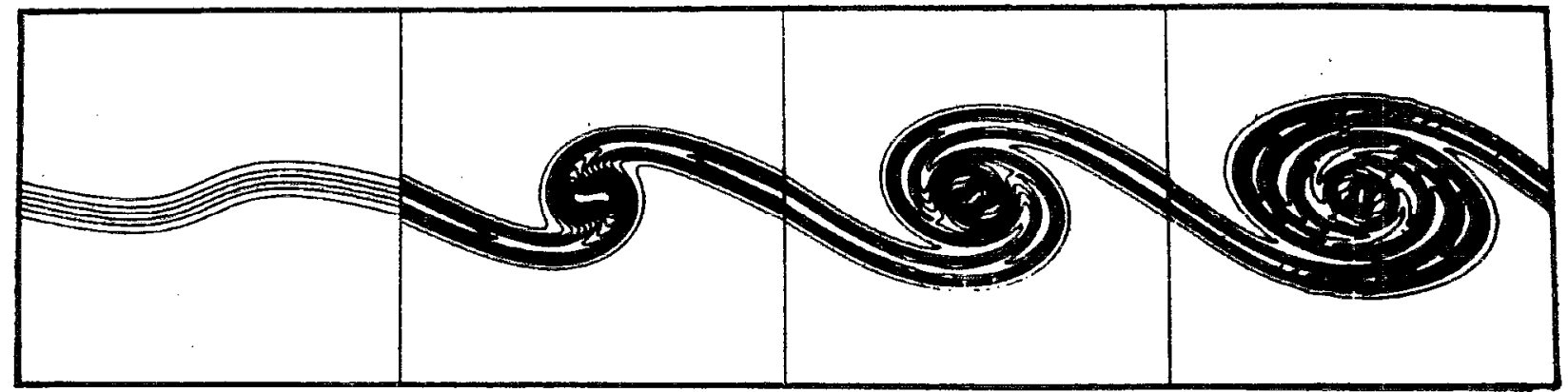

(b)

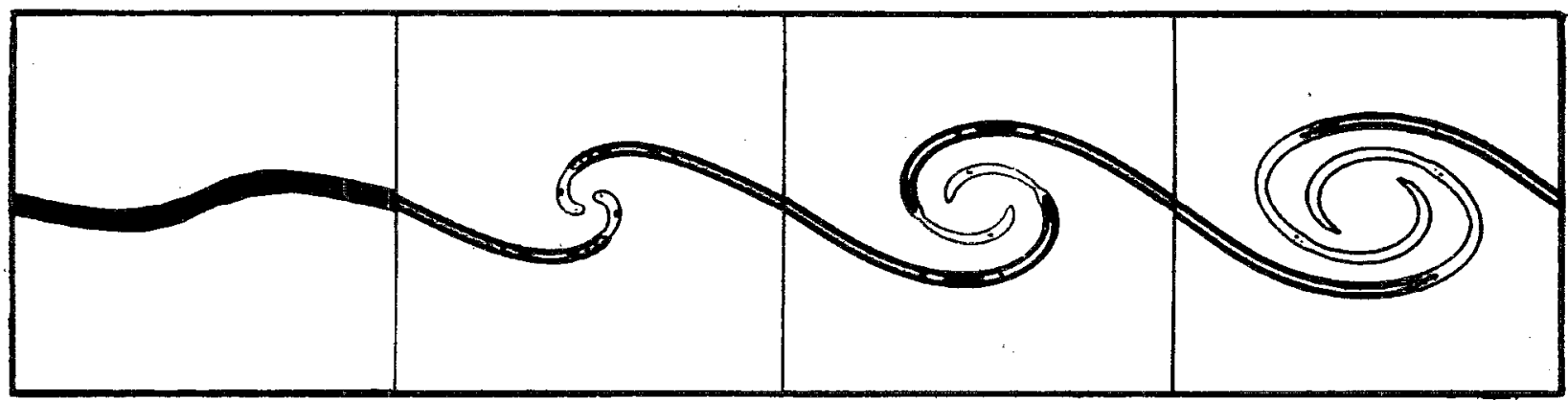

(c)

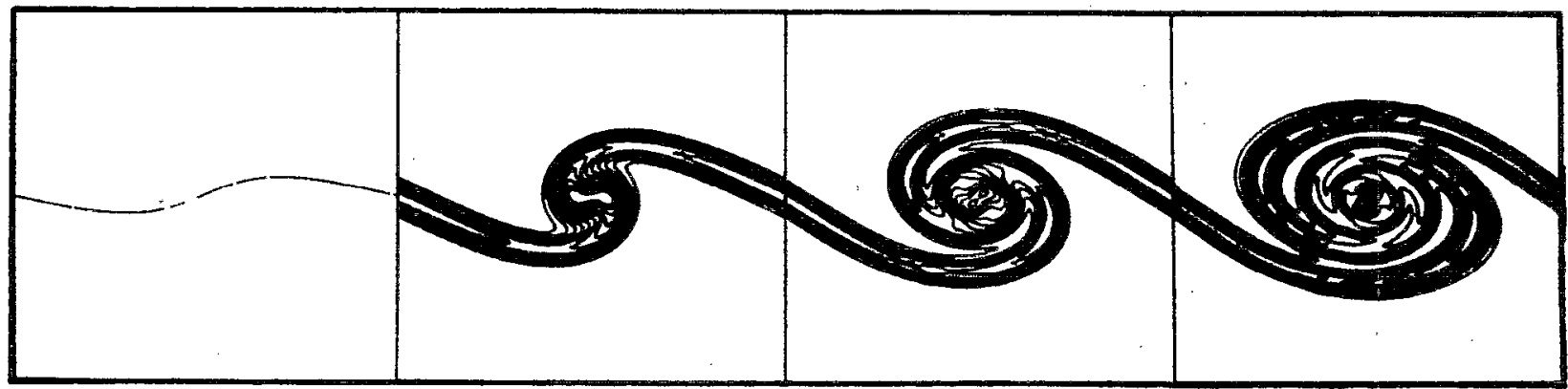

(d)

FIG. 4. Model results as in Fig. 3 but with $\mathrm{Re}=2000$ and $\mathrm{Da}=300$, showing different structure of reacting flow field. Compare with corresponding full finite difference results in Fig. 6. From left to right: $t=0,0.6,1.2$, and 1.8. From top to bottom: (a) The scaled reactant concentration field $c_{A}$, lowest contour level $=0.1$, contour interval $=0.1 ;$ (b) the scaled temperature rise field $T-T_{\circ \times}$, lowest contour level $=1.3$, contour interval $=0.6$; (c) the scaled reaction rate field $\mathrm{Da}\left(c_{A} c_{B}\right) e^{-12 e / n}$, lowest contour level $=0.2$, contour interval $=0.2 ;$ (d) the scaled product concentration field $c_{P}$, lowest contour level $=0.05$, contour interval $=0.05$.

of these moments by coupling the dynamics of the flow field with the mixing and reaction processes.

\section{Layer interactions}

Up to this point the model has considered only isolated diffusion and reaction layers. In general, though, these layers will eventually overlap and interact with one, another, requiring one further approximation in the model. In particular, notice that Eqs. (5)-(7) are linear except for the reaction integrals, suggesting that we might construct the interacting profile from a linear superposition of the two individual profiles as $c_{A}=1-\left(c_{A}^{L}-c_{A}^{R}\right)$ and $c_{B}=\left(c_{B}^{L}+c_{B}^{R}\right)$, where $L$ and $R$ denote the left- and righthand interacting layers. In the absence of chemical reac- 


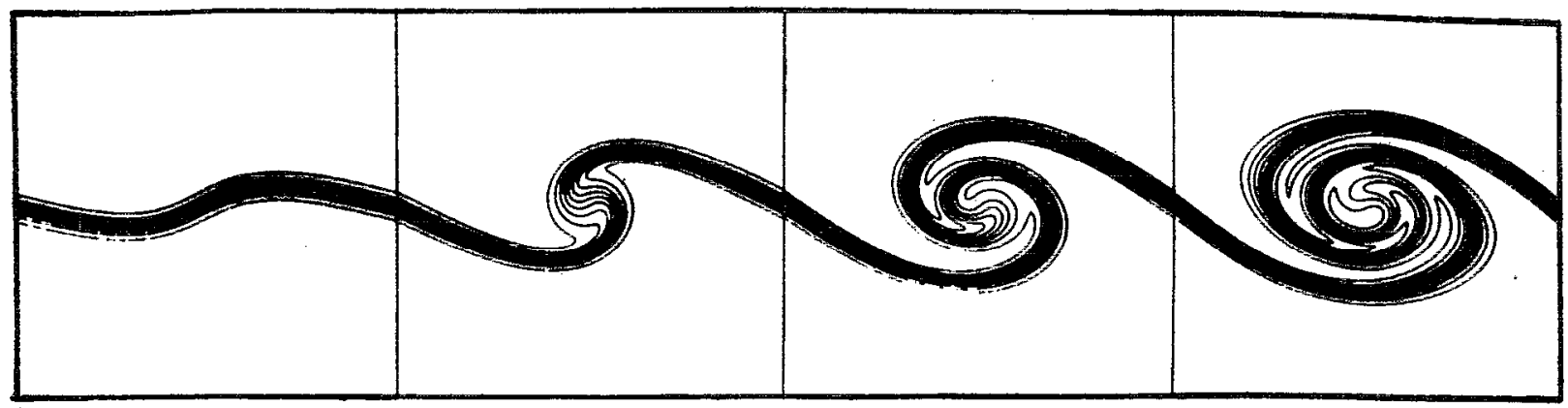

(a)

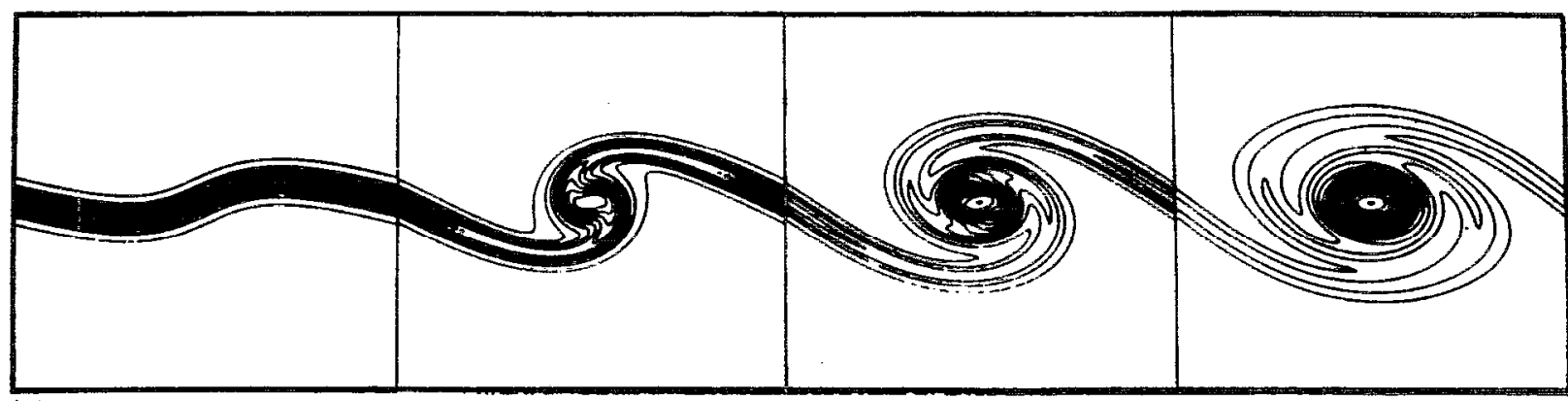

(b)

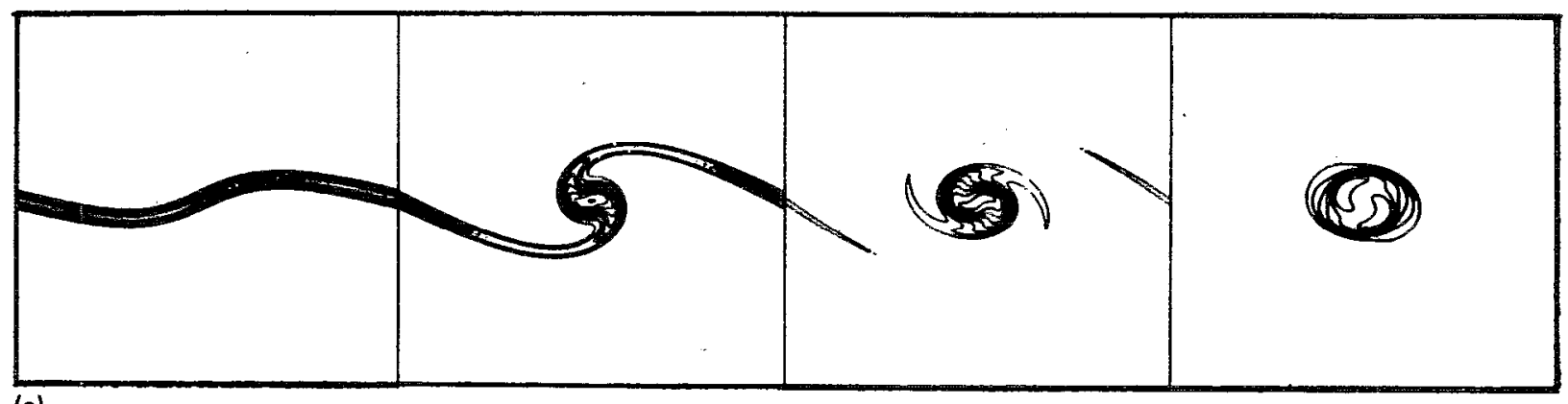

(c)

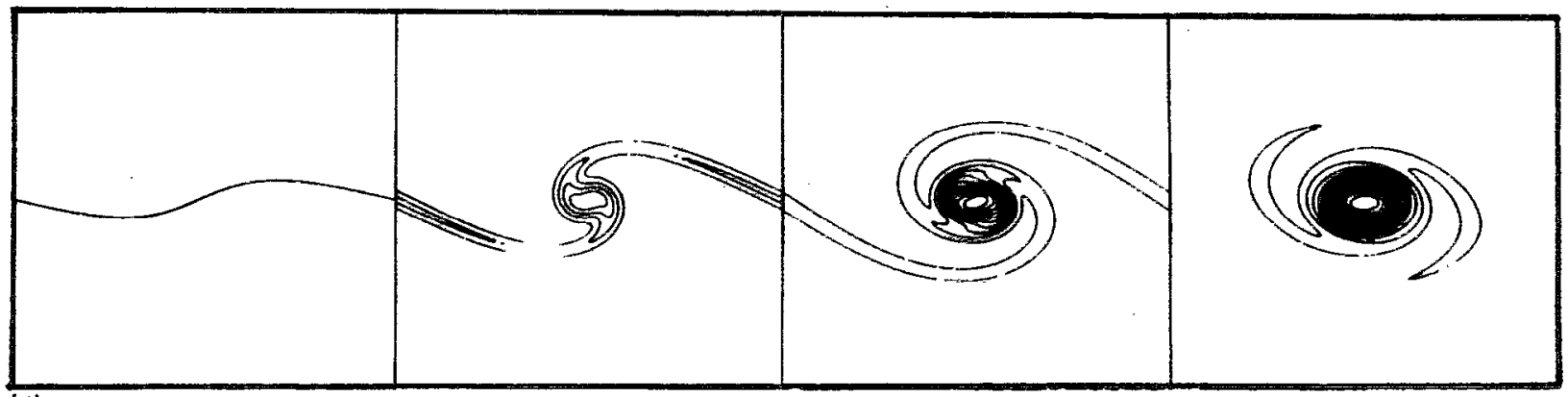

(d)

FIG. 5. Full finite difference simulation results based on Eqs. (2a) and (2b), showing resulting reactant, temperature, reaction rate, and product fields at $\mathrm{Re}=2000$ and $\mathrm{Da}=30$. Compare with corresponding model results in Fig. 3 . Notice that the lowest contour level and contour interval for each field are the same as in Fig. 3. From left to right: $t=0,0.6,1.2$, and 1.8. From top to bottom: (a) The scaled reactant concentration field $c_{A}$; (b) the scaled temperature rise field $T-T_{\alpha} ;$ (c) the scaled reaction rate field $\mathrm{Da}\left(c_{A} c_{B}\right) e^{-1 z_{c} / n} ;$ (d) the scaled product concentration field $c_{P}$.

tions, the moment equations governing the evolution of each of the four component profiles would be completely decoupled. However, when a reaction is present the component profiles for the left- and right-hand layers are coupled through the reaction integrals. To account for this, we will divide the range for these integrals into two parts, one spanning from $-\infty$ to the midpoint between the two layers and affecting only the profiles in the left layer, and the other from the midpoint to $+\infty$ and affecting the profiles on the righthand side layer only. Notice that this approximation is exact for the symmetric reaction that occurs when both layers are at the same conditions. If the layers are dissimilar, then the integration range can in principle be split at a location other than the midpoint between the layers. 


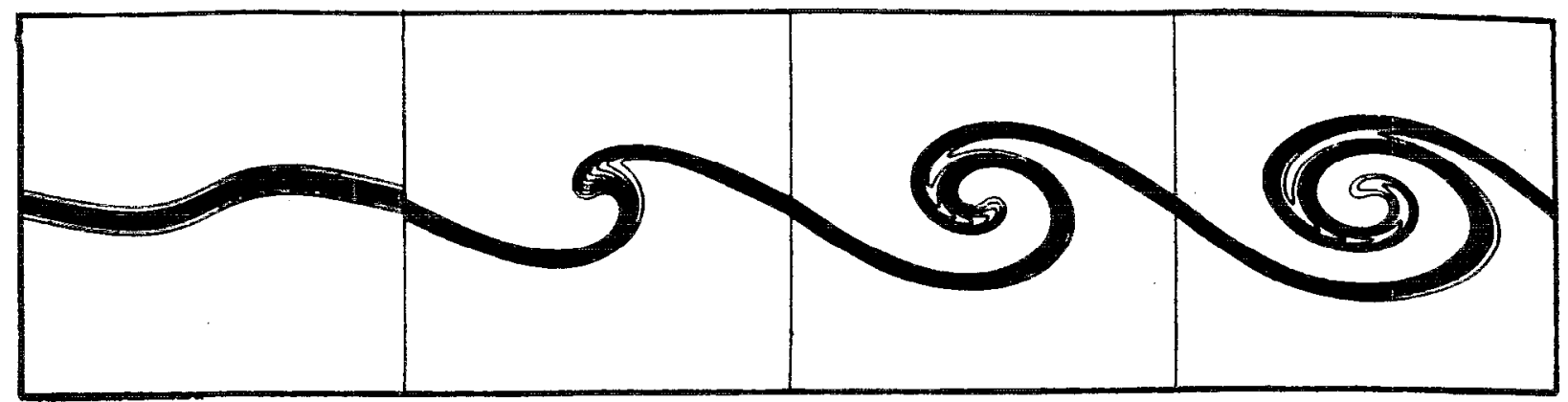

(a)

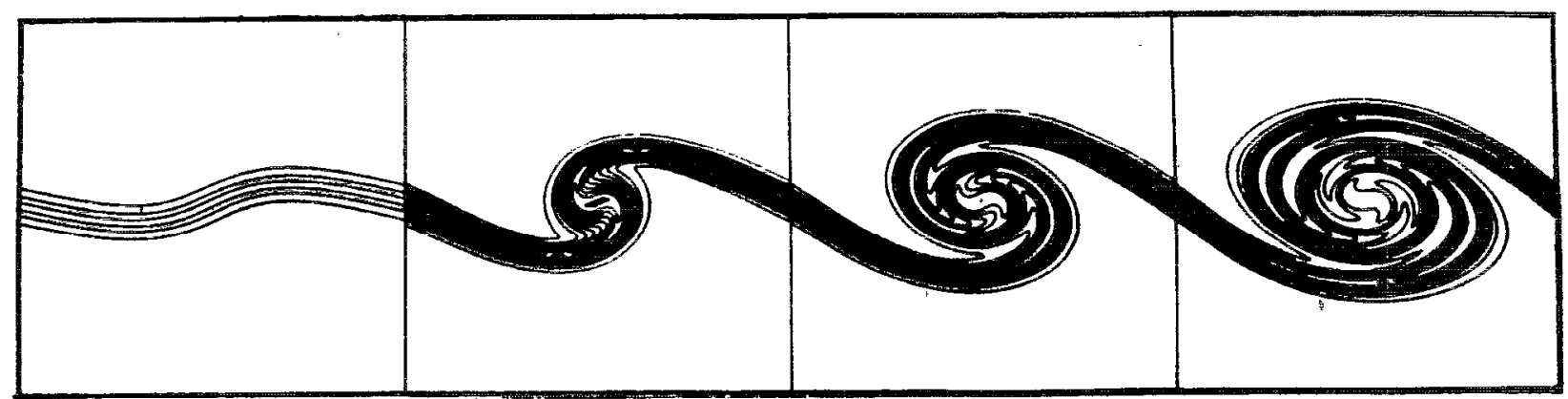

(b)

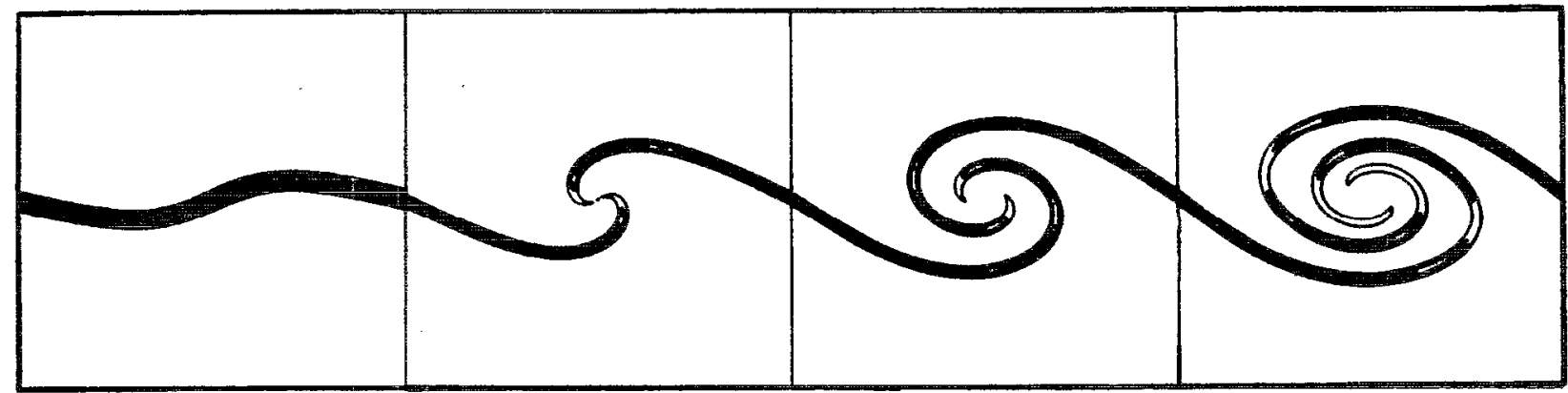

(c)

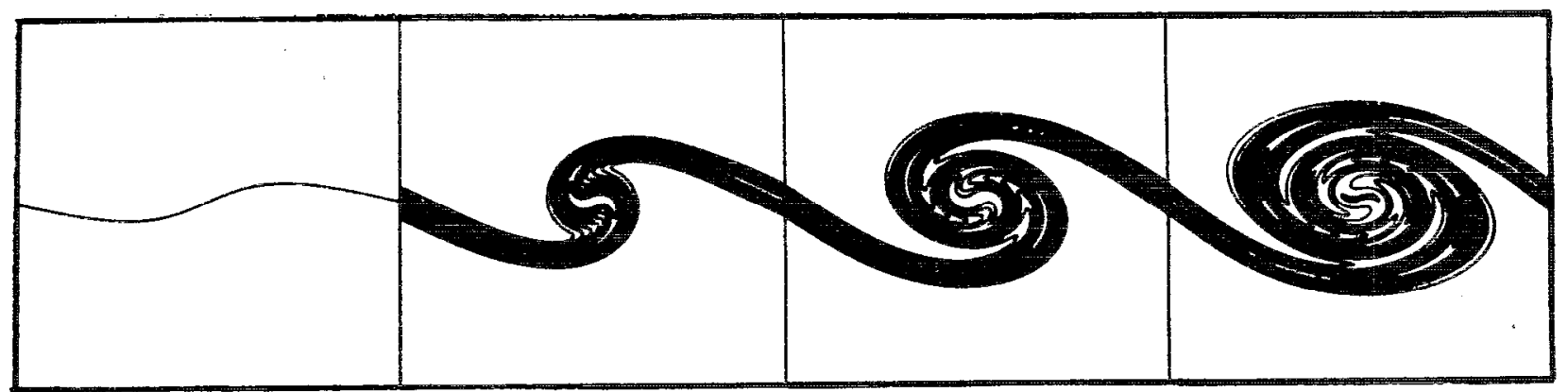

(d)

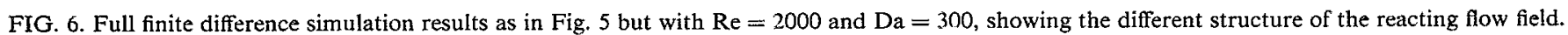

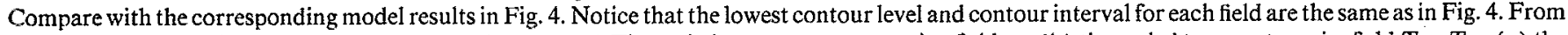

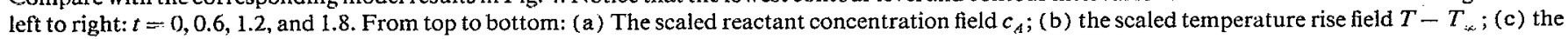
scaled reaction rate field $\mathrm{Da}\left(c_{A} c_{B}\right) e^{-(\mathrm{ze} / T} ;$ (d) the scaled product concentration field $c_{P}$.

\section{SHEAR LAYER RESULTS AND COMPARISONS}

We next apply this model to approximately simulate the molecular mixing and chemical reaction processes in the fundamental roll-up mode of a temporally developing shear layer, and compare the model results with full finite difference calculations of Eqs. (2a) and (2b). Note that the underlying flow field for both the model calculations and the full simulations is obtained here from a finite difference solu- tion of Eq. (1) (also see Ref. 27). The initial thickness of the vorticity layer is $\delta=0.16$, and the layer is given a disturbance with a $5 \%$ amplitude corresponding to the most unstable eigenfunction. For simplicity we have taken all diffusivities to be the same and chosen as reference values the wavelength of the fundamental mode, $L^{*} \equiv \lambda$, and the velocity jump across the layer, $U^{*} \equiv \Delta U$. This gives the Reynolds number as $(\Delta U \cdot \lambda / v)$ and the dimensionless time as 

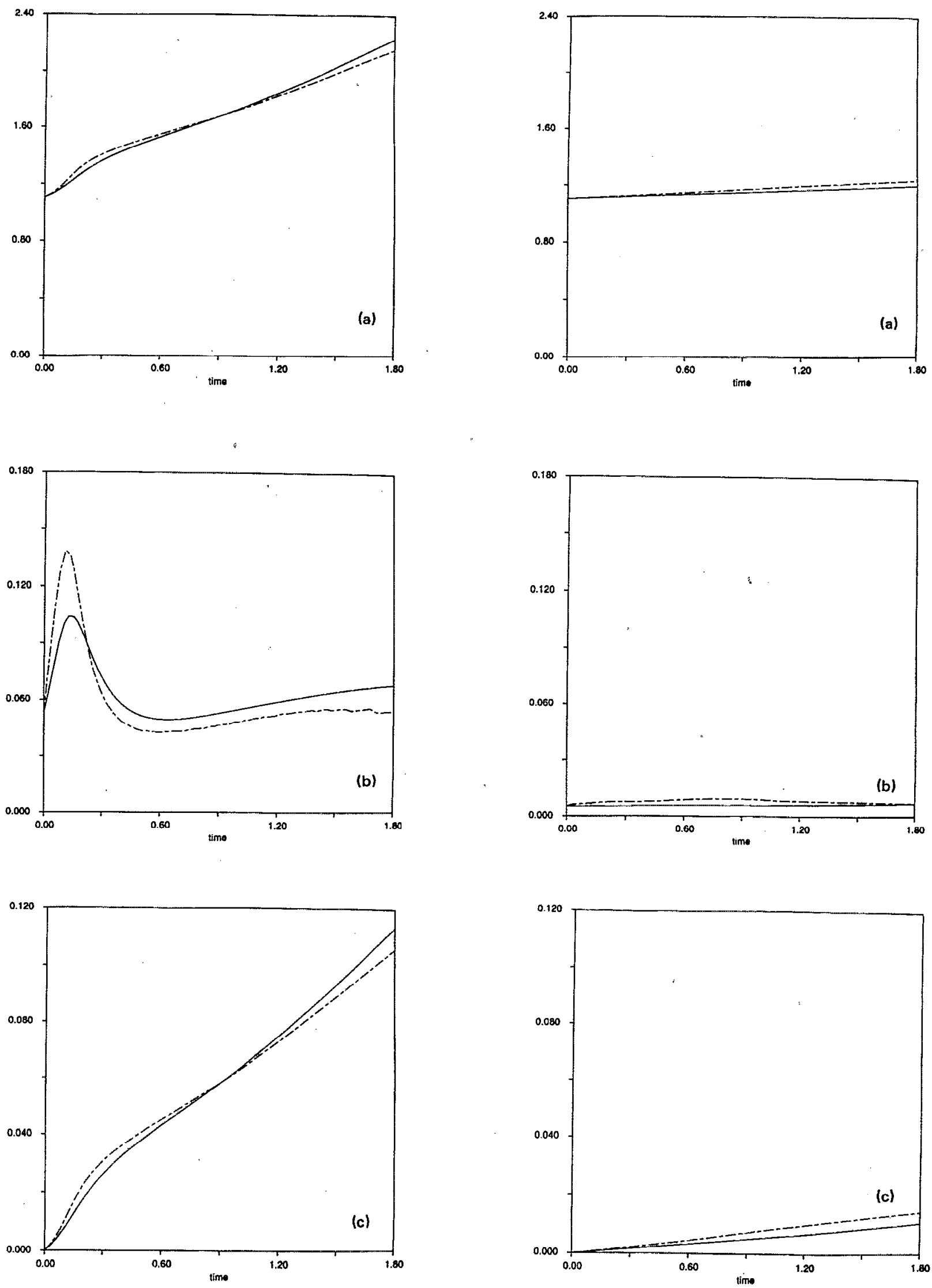

FIG. 7. Comparison of integral quantities obtained from model and finite difference simulation at $\operatorname{Re}=500$ and $\mathrm{Da}=300$. - , finite difference simulations;-- - integral model. (a) The total temperature; (b) the total reaction rate; and (c) the total product.

FIG. 8. Comparison of integral quantities obtained from model and full finite difference simulation at $\operatorname{Re}=2000$ and $\mathrm{Da}=30$. , finite difference simulations; - - integral model. (a) - The total temperature; (b) the total reaction rate; and (c) the total product. 

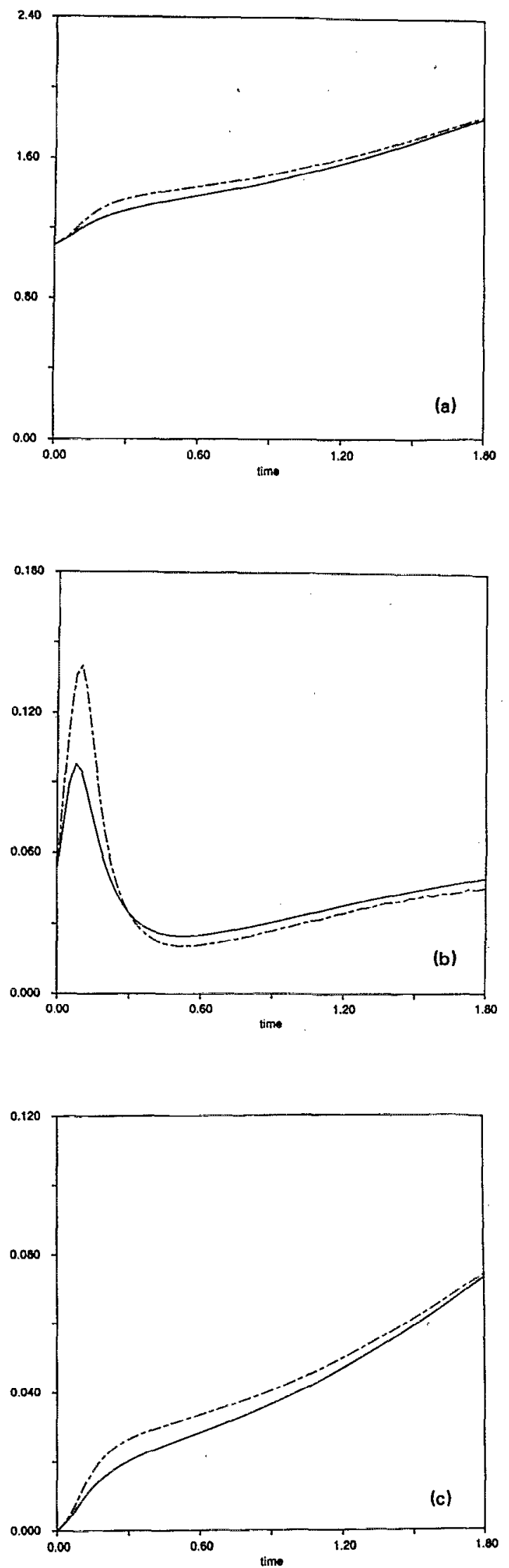

FIG. 9. Comparison of integral quantities obtained from model and full finite diference simulation at $\mathrm{Re}=2000$ and $\mathrm{Da}=300$. - , finite difference simulations; - - , integral model. (a) The total temperature; (b) the total reaction rate; and (c) the total product. $(t \cdot \Delta U / \lambda)$. The temperature is referenced to the free-stream value $T^{*} \equiv T_{\infty}$, and the reactant and product concentrations to $c^{*} \equiv c_{B}^{\infty}$. We have also taken $\mathrm{Sc}=1$ and $\operatorname{Pr}=1$, and have set $\mathrm{Ze}=10$ and $\mathrm{He}=10$ to approximately simulate typical gaseous hydrocarbon chemistry, and for simplicity have set $c_{A}^{\infty}=c_{B}^{\infty}=1$ and $c_{P} \equiv 0$ with $v_{.4}=v_{B}=v_{P}$. The reactions are started at $t=0$ by taking as initial conditions $Q_{0}=10^{-1}$ and $Q_{2}=10^{-4}$ everywhere on the interface that initially separates the two streams.

\section{A. Model results}

Model results for this problem have been calculated at $\operatorname{Re}=500,2000$, and 5000 and at $\mathrm{Da}=30$ and 300. At higher Reynolds and Damköhler numbers, the resolution limitations of the finite difference calculations exceed the grid scale and comparisons are no longer possible. For this reason, we focus first on the results obtained at $\operatorname{Re}=2000$, for which the roll-up of the material interface was shown in Fig. 1. The corresponding model calculations for the reactant concentration field $c_{A}$, the temperature field $T$, the resulting reaction rate field $\mathrm{Da}\left(c_{A} c_{B}\right) e^{-(\mathrm{Ze} / T)}$, and the product concentration field $c_{P}$ are shown in Figs. 3(a)-3(d) for $\mathrm{Da}=30$ and in Figs. 4(a) -4 (d) for $\mathrm{Da}=300$. Each of these fields has been reconstructed from the moment distributions along the interface, with the resulting fields requiring some filtering resulting from the relatively simple reconstruction technique used. Note that the other reactant concentration field $c_{B}$ is here simply the transpose of the $c_{A}$ field shown.

Several features are particularly noteworthy in the results obtained from these model calculations. At the lower Damköhler number, the reaction rate field in Fig. 3(c) suggests that the strain rates in the braids between the vortices are large enough that the reactions there undergo a local extinction phenomenon that is a characteristic of Arrhenius reaction kinetics with large Zel'dovich number (also see Ref. 17). In effect, the reaction there ceases abruptly since the increasing diffusion of heat out of the braids, which results from the steepening of the temperature gradient resulting from the reduction in the layer thickness by the straining, can no longer maintain the temperature high enough to locally sustain the reaction. This is a direct consequence of the strong exponential dependence of the Arrhenius reaction rate on temperature when the Zel'dovich number is relatively large. Once extinction has occurred, the temperature and product in the braids then slowly decay to zero as the continued straining thins the residual temperature and product layer to zero thickness. However, in the vortex cores, where the strain rates are lower, the reaction continues as the reactants gradually diffuse inward from the surrounding areas, producing a slowly decreasing peak reaction rate in the core. The reactant, product, and temperature fields in Fig. 3 also show the resulting effects of these processes in the braids and in the core. In particular, note that reactants are found throughout the core, and the temperature and product.concentrations are relatively low, except in the core. Overall, the key feature at this set of parameter values is that essentially all of the reaction occurs in the core, with little reaction occurring in the spiral arms that lead to the core. 
By comparison, the model results obtained at the higher Damköhler number in Figs. 4(a)-4(d) show a very different set of physical phenomena. In particular, the reaction rate field in Fig. 4(c) shows that, because of the higher kinetic rate at $\mathrm{Da}=300$, the reactions in the braids are now able to sustain themselves and no extinction of the type described above occurs. Essentially all of the reaction occurs in thin layers in the spiral arms leading to the core, with reaction in the core now practically ceasing as the reactants there are consumed [see $c_{A}$ in Fig. 4(a)] and the continuing reactions in the braids do not allow diffusion of much fresh reactants into the core. As a consequence of this different structure in the reaction rate field, the resulting reactant, product and temperature fields in Fig. 4 also show a very different structure than their counterparts in Fig. 3. In particular, the temperature and product fields have a distinct spiral structure with the values remaining high throughout. The reactant field structure in Fig. 4(a) also differs considerably from that in Fig. 3(a).

\section{B. Full simulation comparisons}

We now compare these relatively detailed model results with the results obtained at the same parameter values from the full finite difference simulations of the mixing and reaction processes. In Figs. 5(a)-5(d) we show the resulting reactant concentration field, the temperature field, the reaction rate field, and the product concentration field obtained at $\operatorname{Re}=2000$ and $\mathrm{Da}=30$, and in Figs. 6(a)-6(d) the corresponding results at $\operatorname{Re}=2000$ and $\mathrm{Da}=300$. To allow direct comparisons with the model results, the contour levels are the same in Figs. 3 and 5, and in Figs. 4 and 6.

First we note that essentially the same two distinctly different types of physical phenomena at these two sets of parameter values are seen in the full simulation results, as were seen in the model results. Specifically, in Fig. 5 essentially all of the reaction occurs in the vortex core, with the reactions in the braids straining out, as in Fig. 3. The temperature and product values are low everywhere except in the core, as was also seen in Fig. 3. By comparison, at the higher $\mathrm{Da}$, the full simulation results in Fig. 6 show that relatively little reaction occurs in the core, with essentially all of the reaction taking place in the spiral arms. This can also be seen in the model results in Fig. 4. Moreover, unlike at the lower $\mathrm{Da}$ in Fig. 5, the reactant, temperature, and product fields in Fig. 6 now also show a distinct spiral structure with high values throughout. This is seen also in the model results at the same Da in Fig. 4. Overall, the model results in Figs. 3 and 4 and the full simulation results at the same parameter values in Figs. 5 and 6 are in rather good agreement, despite the widely differing physical phenomena encountered. There are certainly some differences in the fine structure details of the model and simulation results, but at the same parameter values the differences discernible are relatively small in comparison with the much larger differences seen in the structure of the flow at these two different parameter values, suggesting that the model appears to be capable of tracking the evolution of even relatively detailed aspects of the molecular mixing and chemical reaction processes.

\section{INTEGRAL PROPERTIES}

Despite the relatively good detailed agreement seen in Figs. $3-6$ between the model and the full simulation results, it should be kept in mind that the primary objective for which the model was formulated is the prediction of integral properties of the mixing and reaction progress. For this reason, we next examine comparisons of such integral properties obtained from the model and from the full simulations. In each of Figs. 7-9 we show the variation in total temperature, total reaction rate, and total product with time at various Re and Da.

We first examine the results for $\operatorname{Re}=500$ and $\mathrm{Da}=300$ in Fig. 7, for which the detailed structurc of the flow is somewhat similar to that at the higher Re in Figs. 4 and 6. Overall the model follows the simulation results fairly well. Not surprisingly, the biggest differences are in the reaction rate in Fig. 7(b), where both the model and the simulation can be seen to undergo an initial transient before relaxing to an asymptotic evolution. This transient results from the somewhat arbitrary initial conditions chosen to ignite the reactions. In effect, at early times the mixing and reaction progress are still relatively unaffected by the strain along the material interface, and the initial conditions dictate a rapid increase in the reaction rate. Shortly thereafter, the progress of diffusion and reaction within the layer begin to be affected significantly by the strain rate, and the total reaction rate begins to drop. At later times the effects of the initial conditions are lost and the layer relaxes to a state in which its development is governed by balances between the straining of the interface and the diffusion and reaction processes within it. By contrast, at $\mathrm{Re}=2000$ and $\mathrm{Da}=30$ in Fig. 8, the rapid straining out of the reaction in the braids due primarily to the lower $\mathrm{Da}$ results in only a slow increase in the total temperature, reaction rate, and product from their initial conditions. Again the model reproduces this fairly well. The evolution at $\mathrm{Re}=2000$ and $\mathrm{Da}=300$ in Fig. 9 shows a similar initial transient as in Fig. 7, after which the agreement between the model and the full simulation results becomes relatively good. It must be kept in mind that the parameter values here are necessarily low to allow comparisons such as these with corresponding results from full simulations. The underlying thin-layer approximation in the model will likely become more accurate as these parameter values are increased, suggesting that the model results may even become more accurate at the relatively large parameter values that are generally of greatest technical interest.

\section{CONCLUSIONS}

The results presented suggest that the local integral model described here is capable of producing relatively accurate "approximate simulations" of mixing and reaction processes, including highly sensitive nonlinear phenomena such as the local extinction found in large Zel'dovich number Arrhenius kinetics. This conclusion appears to hold even at the relatively low parameter values demanded by the resolution limitations inherent in the full simulations used here to allow a detailed assessment of the model's capabilities. Moreover, the main advantage of the model is that the thin- 
layer approximation on which it is based should become increasingly valid as $\mathrm{Sc}$ and $\mathrm{Da}$ are increased, namely, in precisely the parameter range where full simulations become computationally impractical. The model thus provides an approximate but relatively accurate means for removing the Schmidt number and Damköhler number restrictions on flows involving mixing and chemical reactions.

While we have not conducted a direct study of the reduction in computational time that results from the use of this model for the mixing and reaction parts of the calculations, we note that in calculations of single and interacting layers in Ref. 17, the model was typically between one and two orders of magnitude faster than finite difference simulations at the relatively low parameter values for which full simulations were feasible. As $\mathrm{Sc}$ and $\mathrm{Da}$ are increased, and the associated diffusion and reaction length $\operatorname{scales} \lambda_{D}$ and $\lambda_{R}$ become increasingly finer, such full simulations become increasingly time consuming. The integral nature of the model, on the other hand, leaves the time required for calculations based on it essentially fixed. Thus the real utility of the model can be expected to lie at large parameter values, where the assumptions underlying the model should become increasingly accurate and the reductions in computational time afforded by the model can be expected to become increasingly dramatic.

We also note that extension of the model to more complex sets of reacting species, including non-Arrhenius reactions, as well as to nonuniform transport properties, would appear to be a relatively straightforward matter. A potentially more important point is that the model in its present form addresses only the mixing and reaction processes and does not address the flow field itself, so that the Reynolds number restriction imposed by the length scale $\lambda_{v}$ remains. In this connection, it is interesting to note that an extension of this integral method to the vorticity field is closely related to various vortex blob methods, which typically keep the zeroth moment of the local vorticity profile constant and neglect the effects of strain and diffusion on the vorticity layer thickness (see Ref. 27). It might be possible to formulate a somewhat similar integral method for the vorticity field to take this strain-diffusion balance into account. If coupled with a fast multipole summation scheme, such a local integral method might even prove practical for evolving the flow field underlying the mixing and reaction processes treated here.

\section{ACKNOWLEDGMENTS}

The work described here is being supported by the Gas Research Institute (GRI) under Contract No. .5088-260-
1692, and in part under GRI Contract No. 5087-260-1443 and by the Air Force Office of Scientific Research (AFOSR) under Grant No. AFOSR-89-0541, and with discretionary funds provided by The University of Michigan. Some of the calculations were done on the computers at the San Diego Supercomputer Center, which is sponsored by the National Science Foundation.

${ }^{1}$ J. J. Riley, and R. W. Metcalfe, AIAA Paper No. AIAA-80-0274, 1980.

${ }^{2}$ E. D. Siggia, J. Fluid Mech. 107, 375 (1981).

${ }^{3}$ G. M. Corcos and F. S. Sherman, J. Fluid Mech. 139, 29 (1984).

${ }^{4}$ R. M. Kerr, J. Fluid Mech. 153, 31 (1985).

${ }^{5}$ J. J. Riley, R. W. Metcalfe, and S. A. Orszag, Phys. Fluids 29, 406 (1986).

${ }^{6}$ P. Givi, W.-H. Jou, and R. W. Metcalfe, Proceedings of the 21st International Symposium on Combustion (The Combustion Institute, Pittsburgh, 1986), p. 1251

${ }^{7}$ R. W. Metcalfe, S. A. Orszag, W. E. Brachet, S. Menon, and J. J. Riley, J. Fluid Mech. 184, 207 (1987).

${ }^{8}$ E. S. Oran and J. P. Boris, Numerical Simulation of Reactive Flow (Elsevier, New York, 1987).

${ }^{9}$ V. Eswaran and S. B. Pope, Phys. Fluids 31, 506 (1988).

${ }^{10}$ P. A. McMurtry, J. J. Riley, and R. W. Metcalfe, J. Fluid Mech. 199, 297 (1989).

${ }^{11}$ G. K. Batchelor, J. Fluid Mech. 5, 113 (1959).

${ }^{12}$ G. F. Carrier, F. E. Fendell, and F. E. Marble, SIAM J. Áppl. Math. 28, 463 (1975).

${ }^{13}$ W. J. A. Dahm and K. A. Buch, Proceedings of the 7th Symposium on Turbulent Shear Flows (Stanford University, Stanford, CA, 1989), p. 14.1.1.

${ }^{14}$ W. J. A. Dahm, K. S. Southerland, and K. A. Buch, Proceedings of the 5 th International Symposium on Applications of Laser Techniques to Fluid Mechanics (Instituto Superior Technico, Lisbon, 1990), p. 1.1.1.

${ }^{15}$ W. J. A. Dahm and K. A. Buch, submitted to J. Fluid Mech.

${ }^{16}$ W. J. A. Dahm, K. S. Southerland, and K. A. Buch, Phys. Fluids A 3, 1115 (1991).

${ }^{17}$ G. Tryggvason and W. J. A. Dahm, to appear in Combust. Flame.

${ }^{18}$ F. E. Marble, and J. E. Broadwell, Project SQUID Tech. Rep. No. TRW9-PU, 1977.

${ }^{19}$ C. H. Gibson and P. A. Libby, Combust. Sci. Technol. 8, 29 (1972).

${ }^{20}$ S. K. Liew, K. N. C. Bray, and J. B. Moss, Combust. Sci. Technol. 27, 69 (1981).

${ }^{21}$ N. Peters and F. A. Williams, AIAA J. 21, 423 (1983).

${ }^{22}$ N. Peters and F. A. Williams, Prog. Energy Combust. Sci. 10, 319 (1984).

${ }^{23}$ J. E. Broadwell and R. E. Breidenthal, J. Fluid Mech. 125, 397 (1986).

${ }^{24}$ J. E. Broadwell, and M. G. Mungal, Proceedings of the 22nd Symposium (International) on Combustion (The Combustion Institute, Pittsburgh, 1988), pp. 579-587.

${ }^{25}$ J. E. Broadwell, in Disorder and Mixing, edited by E. Guyon (Kluwer, Boston, 1988)

${ }^{26}$ F. A. Williams, Combustion Theory (Benjamin, Menlo Park, CA, 1985).

${ }^{27}$ G. Tryggvason, W. J. A. Dahm, and K. Sbeih, to appear in ASME J Fluids Eng. 